\title{
Impact of Pre-Radiotherapy and/or Chemoradiotherapy Hemoglobin Level on Response to Treatment in Laryngeal and Hypophayrngeal Squamous Cell Carcinoma
}

\author{
Dina Ragab Diab Ibrahim 1*, Mohamed Saad Hasaballah², Marwa Mohamed El-Begermy², \\ Ahmed Abdel Aziz Ahmed'2, Soha Ahmed Abuelela ${ }^{3}$ \\ ${ }^{1}$ Clinical Oncology Department, Faculty of Medicine, Ain-Shams University, Cairo, Egypt \\ ${ }^{2}$ Ear Nose Throat ENT Department, Faculty of Medicine, Ain-Shams University, Cairo, Egypt \\ ${ }^{3}$ Clinical Pathology Department, Ain-Shams University, Cairo, Egypt \\ Email: *dibrahi1@gmail.com, mshasaballah@hotmail.com, marwabegermy@gmail.com, Ahmedent73@hotmail.com, \\ soha_abuelela@med.asu.edu.eg
}

How to cite this paper: Ibrahim, D.R.D., Hasaballah, M.S., El-Begermy, M.M., Ahmed, A.A.A. and Abuelela, S.A. (2018) Impact of Pre-Radiotherapy and/or Chemoradiotherapy Hemoglobin Level on Response to Treatment in Laryngeal and Hypophayrngeal Squamous Cell Carcinoma. Journal of Cancer Therapy, 9, 362-381.

https://doi.org/10.4236/jct.2018.94033

Received: February 6, 2018

Accepted: April 27, 2018

Published: April 30, 2018

Copyright $\odot 2018$ by authors and Scientific Research Publishing Inc. This work is licensed under the Creative Commons Attribution International License (CC BY 4.0).

http://creativecommons.org/licenses/by/4.0/

(c) (i) Open Access

\begin{abstract}
Background: Anemia is reported by many studies as an important risk factor for poor locoregional disease control and survival in head and neck carcinoma. We aimed to study the frequency and prognostic effect of low hemoglobin $(\mathrm{Hb})$ level in head and neck squamous cell carcinoma (HNSCC) before radiotherapy (RT) and/or chemoradiotherapy (CRT). Material and Methods: We retrospectively studied the charts of 86 patients diagnosed with laryngeal and hypopharyngeal SCC in a university hospital in Cairo, Egypt. Based on the World Health Organization (WHO), anemia was diagnosed in males at $\mathrm{Hb}$ levels $<13 \mathrm{~g} / \mathrm{dl}$ and $<12 \mathrm{~g} / \mathrm{dl}$ in females. We examined the $\mathrm{Hb}$ levels before radiotherapy alone or combined with chemotherapy and its impact on response to treatment and survival. Results: The median age was 56 years. 75/86 (87.2\%) patients were males with performance status1 in 73/86 (84.9\%) patients. The median $\mathrm{Hb}$ level was $13.1 \mathrm{~g} / \mathrm{dl}$. The pre-RT/CRT Hb level in female patients $(\mathrm{n}=11)$ was $<12 \mathrm{~g} / \mathrm{dl}$ in $4 / 11(36.4 \%)$ and $>12 \mathrm{~g} / \mathrm{dl}$ in $7 / 11(63.6 \%)$ patients. Pre-RT/CRT Hb level in male patients was $<13 \mathrm{~g} / \mathrm{dl}$ in $31 / 75$ (41.3\%) and $>13 \mathrm{~g} / \mathrm{dl}$ in 44/75 (58.7\%). Tumor sites were larynx in $77(89.5 \%)$ and hypopharynx in $9(10.5 \%)$ patients. Disease stage 3, 4 was common in 59/86 (68.6\%) patients. Thirty-six (41.8\%) patients had surgery. $13 / 86$ (15.1\%) patients received induction chemotherapy, while 31/86 (36.0\%) had concomitant chemoradiotherapy. Radiotherapy was given in 65/86 (75.58\%) patients. The mean RT dose was 66 Gy. Non-anemic patients had significant higher inci-
\end{abstract}


dence of complete response $(\mathrm{CR})$ to treatment $(\mathrm{p}=0.034) .25 .8 \%$ of male patients with $\mathrm{Hb}<13 \mathrm{~g} / \mathrm{dl}$ had higher incidence of recurrence $(\mathrm{p}=0.036)$ compared to recurrence in $39.5 \%$ of male patients with $\mathrm{Hb}>13 \mathrm{~g} / \mathrm{dl}(\mathrm{p}=0.403)$. Female patients whether anemic or non-anemic had no recurrence $(\mathrm{p}=0.036$ and $p=0.403$ respectively). The median duration of DFS and OS was 6.52 and 9.33 months respectively. Pretreatment $\mathrm{Hb}$ level had statistical significant effect on response to treatment and overall survival, but not disease free survival. Conclusion: Nutritional anemia is common in developing countries. Our results support the positive prognostic effect of $\mathrm{Hb}$ level $>12 \mathrm{~g} / \mathrm{dl}$ and $>13$ $\mathrm{g} / \mathrm{dl}$ before radiation therapy and/or chemoradiotherapy on response to treatment and overall survival but not the disease free survival.

\section{Keywords}

Hemoglobin, Radiotherapy, Chemotherapy, Tumor Hypoxia, Local Control, Survival

\section{Introduction}

Head and neck squamous cell carcinoma HNSCC is a locoregional disease [1], where radiation therapy has a major role in achieving locoregional control whether alone, after surgery or concurrent with chemotherapy [2] [3].

Anemia is a frequent problem in cancer patients that may develop before, during, or after treatment. The incidence of anemia among cancer patients at diagnosis was more than $40 \%$ and $67 \%$ of patients developed anemia during chemotherapy [4]. The incidence of cancer related anemia depends on the type of malignancy, disease stage, duration of the disease, type and intensity of treatment, surgery, and infections [5].

Hemoglobin $\mathrm{Hb}$ level has been identified as one of the strong prognostic factors for tumor control and survival in HNSCC [6]-[13]. Anemia also has negative effect on the quality of life of cancer patients [14]. The prognostic value of anemia was studied at different treatment times in patients with HNSCC. Findings from most studies in HNSCC have consistently found that a low pretreatment $\mathrm{Hb}$ level is a predictor factor of local control and survival after radiotherapy [12]. The incidence of anemia markedly increases when radiation is combined with chemotherapy [15] [16].

Low hemoglobin levels during radiotherapy and post-treatment were also found to affect treatment outcome for HNSCC [17] [18], [11] although not as much investigated as pretreatment anemia. Pre-operative anemia plays a significant role in overall survival [19], where low Hb levels impair tumor oxygenation thus reducing the effect of chemotherapy and radiotherapy [20] [21]. Post-operative anemia was shown to be an independent prognostic factor for local recurrence in locally advanced HNSCC [19] [22].

The range of $\mathrm{Hb}$ levels considered optimal for tumor oxygenation was re- 
ported by most studies to be $12-14 \mathrm{~g} / \mathrm{dL}$ [12] [23]. The etiology of anemia may be multifactorial; the malignancy itself, a complication of the treatment, or the result of an associated comorbidity [24]. Iron deficiency anemia ID is the most common cause of anemia in both solid and hematologic malignancies [25] [26], particularly in low-middle income countries [26]. The prevalence of ID anemia was higher in patients with poor performance status, advanced tumor stage, after chemotherapy, and in persistent or progressive disease [27].

Anemia contributes to hypoxia. Radiation interacts with oxygen to form labile free radicals to kill tumor cells. Anemia decreases the blood oxygen-carrying capacity, and may result in intratumoral hypoxia which compromises the outcome of radiotherapy in various malignancies even the small tumors [15]. Tumor hypoxia and anemia are both independent prognostic indicators of disease free and overall survival in solid tumors, including HNSCC. Hypoxia promotes progression of tumors via hypoxia inducible factor 1 pathway (HIF-1) which in turn up regulates factors that control angiogenesis, apoptosis, and tumor growth [28] [29].

The aim of this study was to investigate the effect of pre-radiotherapy and/or chemotherapy hemoglobin level on local control and survival of patients with laryngeal and hypopharyngeal SCC.

\section{Material and Methods}

We retrospectively reviewed the charts of 86 patients diagnosed with laryngeal and hypopharyrngeal SCC who presented to the head and neck unit at the Department of Clinical Oncology, Ain-Shams University Hospitals during the period from 2009-2013. The patients were referred from the ENT Department, Ain-Shams University Hospitals where they were diagnosed and some of them were operated on. The approval of the Ethical Committee of the Faculty of Medicine, Ain-Shams University was obtained. The patients were considered anemic if pre-RT/CRT hemoglobin was $<12 \mathrm{~g} / \mathrm{dl}$ in females and $<13 \mathrm{~g} / \mathrm{dl}$ in males according to the WHO criteria for anemia [30]. Performance was assessed using the Eastern Cooperative Oncology Group ECOG. Revised Response Evaluation Criteria in Solid Tumors (RECIST) guideline (version 1.1) [31] was used to assess response to treatment as complete response CR or no CR. Acute toxicity during radiotherapy was graded according to the RTOG radiation toxicity criteria [32].

Patient evaluation and staging work-up included complete history, physical examination, endoscopic examination, laboratory and as well as the radiological investigations (chest radiography, computed tomography (CT) and/or magnetic resonance imaging (MRI) of the primary tumor site and the neck). Patients with hemoglobin levels $<9-10 \mathrm{~g} / \mathrm{dL}$ received packed red cell blood transfusion and/or iron supplements. Stage designation was based on the American Joint Committee on Cancer (AJCC) Staging Criteria $7^{\text {th }}$ Edition [33]. CT neck was done 8 - 10 weeks post treatment to assess response, and then repeated during 
follow-up.

\section{Statistical Analysis}

It was done using the Statistical Package for the Social Sciences (SPSS) version 20. The qualitative data were presented as number and percentages while quantitative data were presented as mean, standard deviations and ranges when their distribution was parametric while non-parametric data were presented as median with interquartile range (IQR). Comparison of categorical variables was carried by using Chi-square test. DFS was calculated from date of start of radiotherapy and/or chemoradiotherapy till date of recurrence. OS was calculated from date of pathological diagnosis till date of last follow-up or death. The confidence interval was set to $95 \%$. P-value $<0.05$ was considered statistical significance.

\section{Results}

Eighty-six patients pathologically diagnosed with laryngeal and hypopharyngeal SCC were included in the study. The mean age was 56 years (range $27-80$ ). The cohort had more male patients, 75/86 (87.2\%) and ECOG performance status (PS) 1in 73/86 (84.9\%) patients. Laryngeal SCC (supraglottic SCC in 42 patients, glottic SCC in 33 patients and subglottic SCC in 2 patients) was the commonest tumor site in 77/86 (89.5\%) patients. Tumor characteristics showed predominance of T3 in 33/86 (38.4\%) patients, and N0 in 51/86, (59.3\%) patients. Only 3 patients (3.5\%) had metastatic disease (M1) from the start and 42/86 (48.8\%) patients had stage 4 . The median pre-RT/CRT Hb was $13.1 \mathrm{~g} / \mathrm{dl}$ (range 11.9 - 14.2). Most of female patients 7/11 (63.6\%) had $\mathrm{Hb}>12 \mathrm{~g} / \mathrm{dl}$, and most male patients 44/75 (58.7\%) had $\mathrm{Hb}>13 \mathrm{~g} / \mathrm{dl}$ (Table 1). By the end of the follow-up duration of 9.33 months, 59 patients (86.6\%) were alive and 27 (31.4\%) patients were dead.

Table 2 shows the summary of treatment. Surgery was the primary treatment in 36/86 (41.8\%) patients; total laryngectomy and neck dissection was the commonest type performed in $15 / 86(17.4 \%)$ patients. Most of the patients $65 / 86$ (76\%) received some form of radiotherapy (RT), where RT (adjuvant or definitive) was administered in 34/86 (39.53\%) patients while concomitant chemoradiotherapy (CCRT) (adjuvant or definitive) was indicated in 31/86 (36.05\%) patients. Fractionation was conventional (200 cGy/fraction) in most of the patients, and hypofractionated (2.25 cGy/fraction) in 4 glottic SCC patients. The mean radiation dose was $66 \mathrm{~Gy}$. Mucositis during radiotherapy occurred in $52 / 65(80 \%)$ of irradiated patients. The Indications of chemotherapy included induction, and concurrent with radiotherapy. Only 13/86 (15.1\%) patients received induction chemotherapy. The induction regimens included TP (taxotere/cisplatin) q 21 days for 3 cycles, TPF (taxotere, cisplatin, 5-fluorouracil 5 FU) q 21 days for 3 cycles, and taxotere/carboplatin q 21 days for 3 cycles. 31/86 (36.0\%) patients received concomitant chemotherapy; cisplatin $100 \mathrm{mg} / \mathrm{m}^{2}$, weekly cisplatin $30-50 \mathrm{mg} / \mathrm{m}^{2}$ or weekly carboplatin (AUC 1.5). 
Table 1. Patient and tumor characteristics.

\begin{tabular}{|c|c|}
\hline \multicolumn{2}{|l|}{ Age } \\
\hline Median (IQR) & $56(50-66)$ \\
\hline Range & $27-80$ \\
\hline \multicolumn{2}{|l|}{ ECOG } \\
\hline 0 & $1(1.2 \%)$ \\
\hline 1 & $73(84.9 \%)$ \\
\hline 2 & $12(14.0 \%)$ \\
\hline \multicolumn{2}{|l|}{ Gender } \\
\hline Male & $75(87.2 \%)$ \\
\hline female & $11(12.8 \%)$ \\
\hline \multicolumn{2}{|l|}{ Site } \\
\hline Larynx & 77 (89.5\%) \\
\hline Hypopharynx & $9(10.5 \%)$ \\
\hline \multicolumn{2}{|l|}{ Grade } \\
\hline 1 & $14(16.3 \%)$ \\
\hline 2 & $61(70.9 \%)$ \\
\hline 3 & $11(12.8 \%)$ \\
\hline \multicolumn{2}{|l|}{ Tumor T-stage } \\
\hline 1 & $11(12.8 \%)$ \\
\hline 2 & $26(30.2 \%)$ \\
\hline 3 & $33(38.4 \%)$ \\
\hline 4 & $16(18.6 \%)$ \\
\hline \multicolumn{2}{|l|}{ Nodal N-stage } \\
\hline 0 & $51(59.3 \%)$ \\
\hline 1 & $5(5.8 \%)$ \\
\hline 2 & $26(30.2 \%)$ \\
\hline 3 & $4(4.7 \%)$ \\
\hline \multicolumn{2}{|l|}{ AJCC stage } \\
\hline 1 & $11(12.8 \%)$ \\
\hline 2 & $16(18.6 \%)$ \\
\hline 3 & $17(19.8 \%)$ \\
\hline 4 & $42(48.8 \%)$ \\
\hline \multicolumn{2}{|l|}{ Pretreatment hemoglobin $(\mathrm{g} / \mathrm{dl})$} \\
\hline Median (IQR) & $13.1(11.9-14.2)$ \\
\hline$<13$ (male) & $31(41.3 \%)$ \\
\hline$>13$ (male) & $44(58.7 \%)$ \\
\hline$<12$ (female) & $4(36.4 \%)$ \\
\hline$>12$ (female) & $7(63.6 \%)$ \\
\hline Median follow-up duration & $9.33(4.2-17.7)$ \\
\hline
\end{tabular}

ECOG: Eastern Cooperative Oncology Group, AJCC: American Joint Committee on Cancer.

Table 2. Treatment characteristics.

\begin{tabular}{cccc}
\hline & No. & $\%$ \\
\hline Surgery & & \\
Total laryngectomy and ND & 15 & $17.4 \%$ \\
Total laryngectomy & 13 & $15.1 \%$ \\
Other & 8 & $9.3 \%$ \\
\hline
\end{tabular}




\section{Continued}

\begin{tabular}{|c|c|c|}
\hline No & 44 & $51.2 \%$ \\
\hline NA & 6 & $7.0 \%$ \\
\hline \multicolumn{3}{|l|}{ Induction chemotherapy } \\
\hline No & 65 & $75.6 \%$ \\
\hline NA & 8 & $9.3 \%$ \\
\hline TPF & 6 & $7.0 \%$ \\
\hline $\mathrm{TP}$ & 3 & $3.5 \%$ \\
\hline Cisplatin/5 Fluorouracil & 4 & $4.7 \%$ \\
\hline Carboplatin/5 Fluorouracil & 0 & $0.0 \%$ \\
\hline \multicolumn{3}{|l|}{ Concurrent chemotherapy } \\
\hline No & 47 & $54.7 \%$ \\
\hline NA & 8 & $9.3 \%$ \\
\hline Cisplatin & 15 & $17.4 \%$ \\
\hline Carboplatin & 16 & $18.6 \%$ \\
\hline \multicolumn{3}{|l|}{ Radiotherapy RT } \\
\hline No & 13 & $15.1 \%$ \\
\hline NA & 8 & $9.3 \%$ \\
\hline Alone (Adjuvant-definitive) & 34 & $39.5 \%$ \\
\hline Concurrent (Adjuvant-definitive) & 31 & $36.0 \%$ \\
\hline \multicolumn{3}{|l|}{ Acute RT toxicity } \\
\hline No & 17 & $19.8 \%$ \\
\hline NA & 7 & $8.1 \%$ \\
\hline Mucositis & 52 & $60.5 \%$ \\
\hline Skin reaction & 10 & $11.6 \%$ \\
\hline
\end{tabular}

NA: Not Available, ND: Nodal Dissection.

Table 3 depicts the response to treatment where 25/86 (29.1\%) patients developed local recurrence. None of the patients developed distant metastases till the end of the study period. After a median follow-up duration of 9.33 months, the median DFS of the patients was 6.52 months while the median OS was 9.33 months.

We studied the prognostic factors affecting response to treatment complete response (CR) versus no $\mathrm{CR}$ as shown in (Table 4). The treatment data were reported for $80 / 86$ patients as $6 / 86$ (7\%) patients' charts were incomplete. $46 / 86$ (53.5\%) patients achieved CR whereas 34/86 (39.5\%) patients had no CR. Significant factors were T-stage, $\mathrm{N}$-stage, gender and pre-RT/CRT Hb level. The incidence of CR was ( $\mathrm{p}=0.010)$ higher in male (95.7\%) than female patients $(4.3 \%)$. Both CR and no CR occurred more in patients with pre-RT/CRT Hb levels $>13$ and $>12 \mathrm{~g} / \mathrm{dl}(\mathrm{p}=0.034) .41 / 46(89.1 \%)$ patients with laryngeal SCC responded 
to treatment by CR versus 24/34 (70.6\%) patients who had no CR ( $\mathrm{p}=0.036$ ). On the other hand hypopharyngeal SCC tumors had higher rates of no CR 10/34 $(29.4 \%)$ than CR in 5/46 $(10.9 \%)(\mathrm{p}=0.036)$ patients. AJCC Stage 3,4 responded to treatment by no CR in $85.3 \%$ of patients, whereas stages 1,2 achieved more CR in $45.7 \%$ of patients ( $\mathrm{p}=0.003$ ).

Analysis of the prognostic factors affecting DFS and OS showed that age, gender, performance status at presentation, N-stage and pre-RT/CRT Hb level significantly influenced OS, but none of the studied factors were significantly predictive of DFS (Table 5, Figures 1-5). Patients $<56$ years had longer median OS of 32.567 months than patients $>56$ years who survived for a median of 15.900 months $(\mathrm{p}=0.039)$. Median OS of male and female patients was 26.567, and 11.433 months respectively $(\mathrm{p}=0.000)$. Patients with N0 and N1 disease had median OS 28.290 months, and 18.227 months respectively $(\mathrm{p}=0.031)$. The median DFS of patients with pre-RT/CRT anemia versus no anemia was comparable 19.442, 19.496 months respectively $(\mathrm{p}=0.233)$. Non-anemic patients had significantly better median OS compared to anemic patients (44.134 and 38 months respectively, $\mathrm{p}=0.000)$.

Table 3. Treatment results.

\begin{tabular}{cc}
\hline Variable & Frequency \\
\hline Complete response (CR) & $46(53.5 \%)$ \\
No complete response (No CR) & $34(39.5 \%)$ \\
NA & $6(7.0 \%)$ \\
Recurrence & $25(29.1 \%)$ \\
Status at last follow-up visit & $59(68.6 \%)$ \\
Alive & $27(31.4 \%)$ \\
Dead & $6.52(3.27-12.37)$ \\
Disease free survival (DFS) & \\
median (IQR) & $9.33(4.2-17.67)$ \\
Overall survival (OS) & median (IQR)
\end{tabular}

IQR: Inter Quartile Range.

Table 4. Prognostic factors of response to treatment.

\begin{tabular}{|c|c|c|c|c|c|c|c|c|}
\hline & & \multicolumn{2}{|c|}{$\begin{array}{c}\text { CR } \\
(n=46)\end{array}$} & \multicolumn{2}{|c|}{$\begin{array}{c}\text { No } C R \\
(n=34)\end{array}$} & \multirow[t]{2}{*}{ Test value } & \multirow{2}{*}{ p-value } & \multirow[t]{2}{*}{ Significanc } \\
\hline & & No. & $\%$ & No. & $\%$ & & & \\
\hline \multirow[b]{2}{*}{ Age } & $<56$ years & 19 & $41.3 \%$ & 18 & $52.9 \%$ & \multirow[b]{2}{*}{1.065} & \multirow[b]{2}{*}{0.302} & \multirow[b]{2}{*}{ NS } \\
\hline & $>56$ years & 27 & $58.7 \%$ & 16 & $47.1 \%$ & & & \\
\hline \multirow[b]{2}{*}{ Gender } & Male & 44 & $95.7 \%$ & 26 & $76.5 \%$ & \multirow[b]{2}{*}{6.577} & \multirow[b]{2}{*}{0.010} & \multirow[b]{2}{*}{ S } \\
\hline & Female & 2 & $4.3 \%$ & 8 & $23.5 \%$ & & & \\
\hline \multirow{2}{*}{ Site of the tumor } & Larynx & 46 & $100.0 \%$ & 26 & $76.5 \%$ & \multirow{2}{*}{12.026} & \multirow{2}{*}{0.001} & \multirow{2}{*}{ HS } \\
\hline & Hypopharynx & 0 & $0.0 \%$ & 8 & $23.5 \%$ & & & \\
\hline T-staging & $\mathrm{T} 1+2$ & 25 & $54.3 \%$ & 10 & $29.4 \%$ & 4.94 & 0.026 & S \\
\hline
\end{tabular}




\section{Continued}

\begin{tabular}{|c|c|c|c|c|c|c|c|c|}
\hline & $\mathrm{T} 3+4$ & 21 & $45.7 \%$ & 24 & $70.6 \%$ & & & \\
\hline \multirow{2}{*}{$\mathrm{N}$-stage } & No & 34 & $73.9 \%$ & 13 & $38.2 \%$ & \multirow{2}{*}{10.269} & \multirow{2}{*}{0.001} & \multirow{2}{*}{ HS } \\
\hline & $\mathrm{N} 1,2,3$ & 12 & $26.1 \%$ & 21 & $61.8 \%$ & & & \\
\hline \multirow{2}{*}{ Stage } & $1+2$ & 21 & $45.7 \%$ & 5 & $14.7 \%$ & \multirow{2}{*}{8.534} & \multirow{2}{*}{0.003} & \multirow{2}{*}{ HS } \\
\hline & $3+4$ & 25 & $54.3 \%$ & 29 & $85.3 \%$ & & & \\
\hline \multirow{4}{*}{ Pre -RT/CRT Hb level } & $<13$ & 19 & $41.3 \%$ & 10 & $29.4 \%$ & \multirow{4}{*}{8.689} & \multirow{4}{*}{0.034} & \multirow{4}{*}{ S } \\
\hline & $<12$ & 0 & $0.0 \%$ & 3 & $8.8 \%$ & & & \\
\hline & $>13$ & 25 & $54.3 \%$ & 16 & $47.1 \%$ & & & \\
\hline & $>12$ & 2 & $4.4 \%$ & 5 & $14.7 \%$ & & & \\
\hline
\end{tabular}

Table 5. Prognostic factors of DFS and OS.

\begin{tabular}{|c|c|c|c|c|c|c|c|c|}
\hline & & No. & Mean & SD & $\begin{array}{l}\text { OS (median) } \\
\text { (months) }\end{array}$ & p-value & $\begin{array}{l}\text { DFS (median) } \\
\quad \text { (months) }\end{array}$ & p-value \\
\hline \multirow{2}{*}{ Age } & $<56$ years & 41 & 13 & 2 & 32.567 & \multirow{2}{*}{0.039} & 25.000 & \multirow{2}{*}{0.785} \\
\hline & $>56$ years & 45 & 12.9 & 1.9 & 15.900 & & 20.000 & \\
\hline \multirow{2}{*}{ Gender } & Male & 75 & 13.1 & 1.9 & 26.567 & \multirow{2}{*}{0.000} & 9.584 & \multirow{2}{*}{0.066} \\
\hline & Female & 11 & 11.7 & 1.8 & 11.433 & & 9.545 & \\
\hline \multirow{2}{*}{ Performance at presentation } & 1 & 73 & 13.1 & 1.9 & 26.567 & \multirow{2}{*}{0.002} & 20.000 & \multirow{2}{*}{0.120} \\
\hline & 2 & 12 & 11.9 & 1.9 & 11.567 & & - & \\
\hline \multirow{2}{*}{ Treatment } & $\mathrm{RT}$ & 34 & 13.4 & 1.6 & 24.567 & \multirow{2}{*}{0.907} & 20.200 & \multirow{2}{*}{0.731} \\
\hline & CCRT & 31 & 12.8 & 2.1 & 18.667 & & 17.200 & \\
\hline \multirow[b]{2}{*}{ RT dose } & $<66$ & 45 & 12.8 & 1.8 & 24.567 & \multirow[b]{2}{*}{0.569} & - & \multirow[b]{2}{*}{0.131} \\
\hline & $>66$ & 20 & 13.6 & 1.9 & 26.567 & & 20.200 & \\
\hline \multirow[b]{2}{*}{ Surgery } & Yes & 36 & 13.1 & 1.9 & 25.000 & \multirow[b]{2}{*}{0.059} & 20.000 & \multirow{2}{*}{0.724} \\
\hline & No & 44 & 12.8 & 2 & 14.700 & & 13.200 & \\
\hline \multirow{3}{*}{ Grade } & 1 & 14 & 13.5 & 1.9 & 36.571 & \multirow{3}{*}{0.155} & 30.946 & \multirow{3}{*}{0.306} \\
\hline & 2 & 61 & 12.9 & 2 & 22.175 & & 20.685 & \\
\hline & 3 & 11 & 12.6 & 1.9 & 22.447 & & 20.028 & \\
\hline \multirow{2}{*}{ T-stage } & 1,2 & 37 & 13.5 & 2 & 28.790 & \multirow{2}{*}{0.554} & 26.621 & \multirow{2}{*}{0.299} \\
\hline & 3,4 & 49 & 12.5 & 1.8 & 23.530 & & 20.940 & \\
\hline \multirow{2}{*}{ N-stage } & 0 & 51 & 13.2 & 1.9 & 28.290 & \multirow{2}{*}{0.031} & 25.228 & \multirow{2}{*}{0.647} \\
\hline & 1 & 35 & 12.6 & 1.9 & 18.227 & & 17.824 & \\
\hline \multirow{2}{*}{ M } & 0 & 83 & 13 & 2 & 23.592 & \multirow{2}{*}{0.365} & 22.048 & \multirow{2}{*}{0.735} \\
\hline & 1 & 3 & 13 & 0.8 & 27.522 & & 23.889 & \\
\hline \multirow{2}{*}{ AJCC stage } & 1,2 & 27 & 14.01 & 1.7 & 32.019 & 0297 & 33.64 & 0085 \\
\hline & 3,4 & 59 & 12.48 & 1.9 & 23.004 & 0.297 & 20.15 & 0.083 \\
\hline & $<12$ & 4 & 9.9 & 1.9 & 11.433 & & 11.292 & \\
\hline Dep DT/CDT Hb low & $<13$ & 31 & 11.4 & 1.3 & 26.567 & 00000 & 8.150 & 0223 \\
\hline 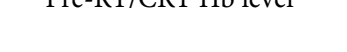 & $>12$ & 8 & 12.8 & 0.5 & 11.567 & 0.000 & 8.925 & 0.253 \\
\hline & $>13$ & 43 & 14.4 & 1.1 & 32.567 & & 10.571 & \\
\hline
\end{tabular}




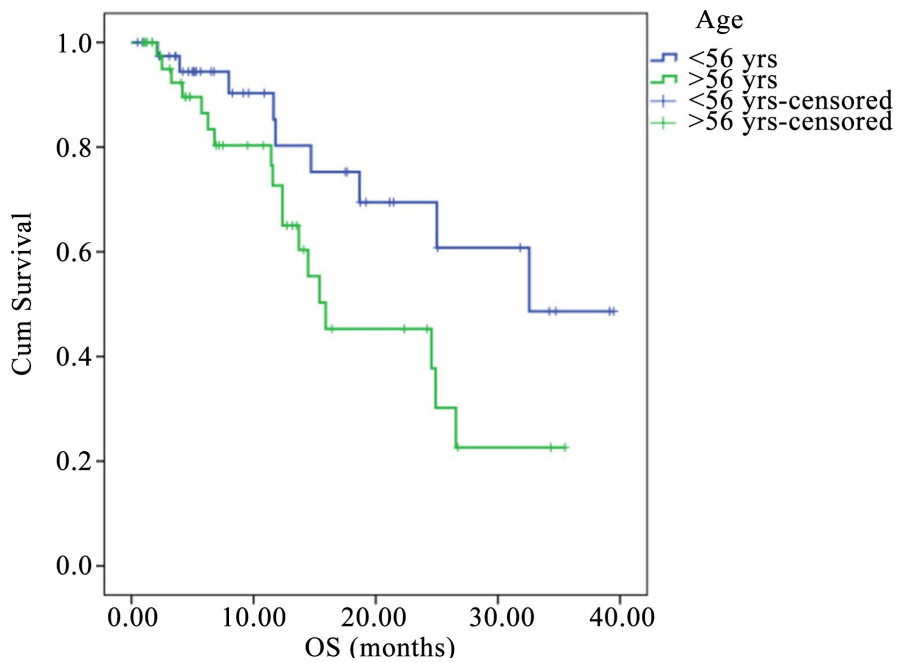

Figure 1. Effect of age on OS.

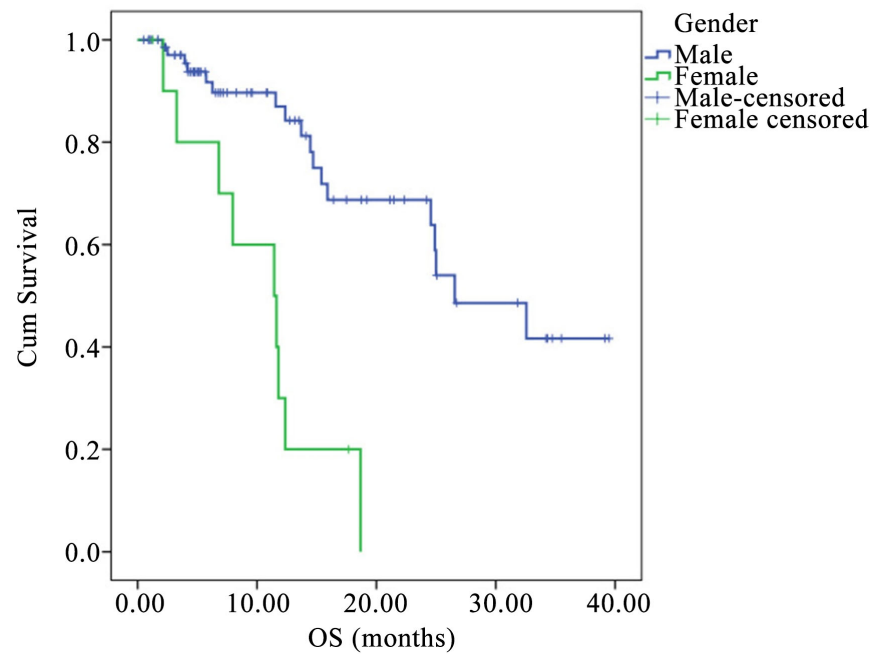

Figure 2. Effect of gender on OS $(n=86)$.

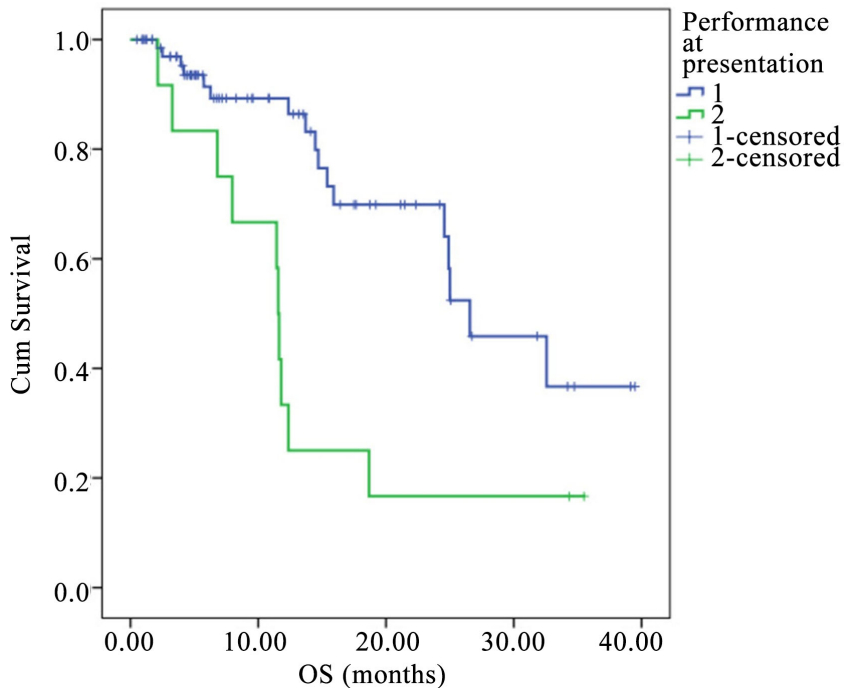

Figure 3. Effect of performance on OS $(n=86)$. 


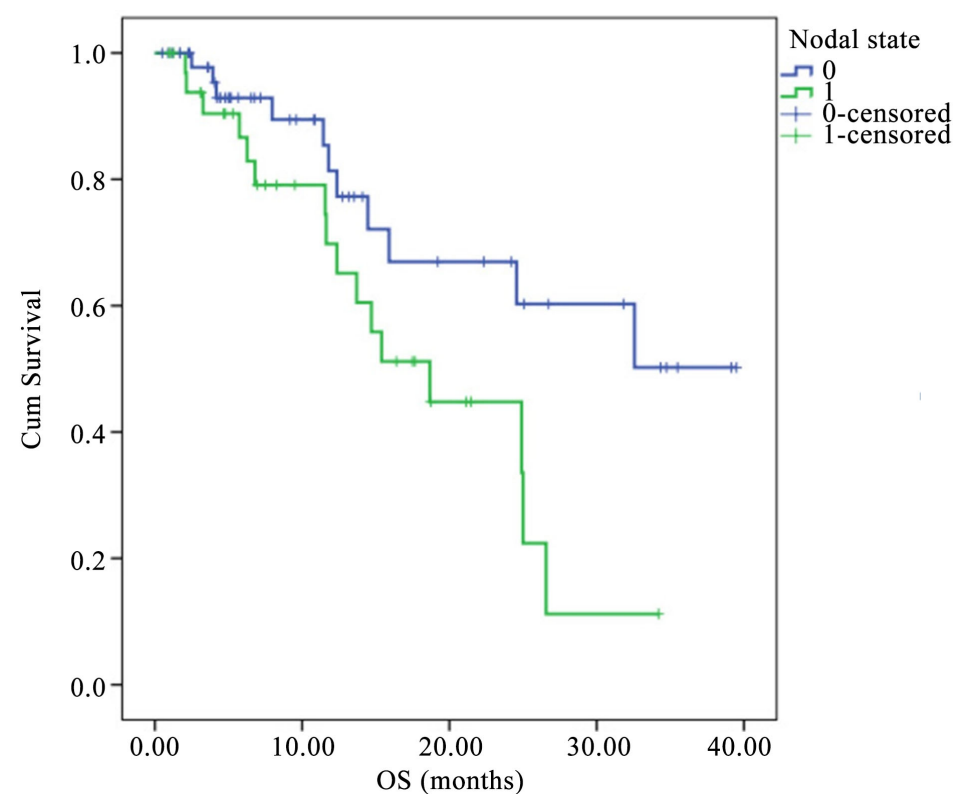

Figure 4. Effect of nodal stage on OS $(n=86)$.

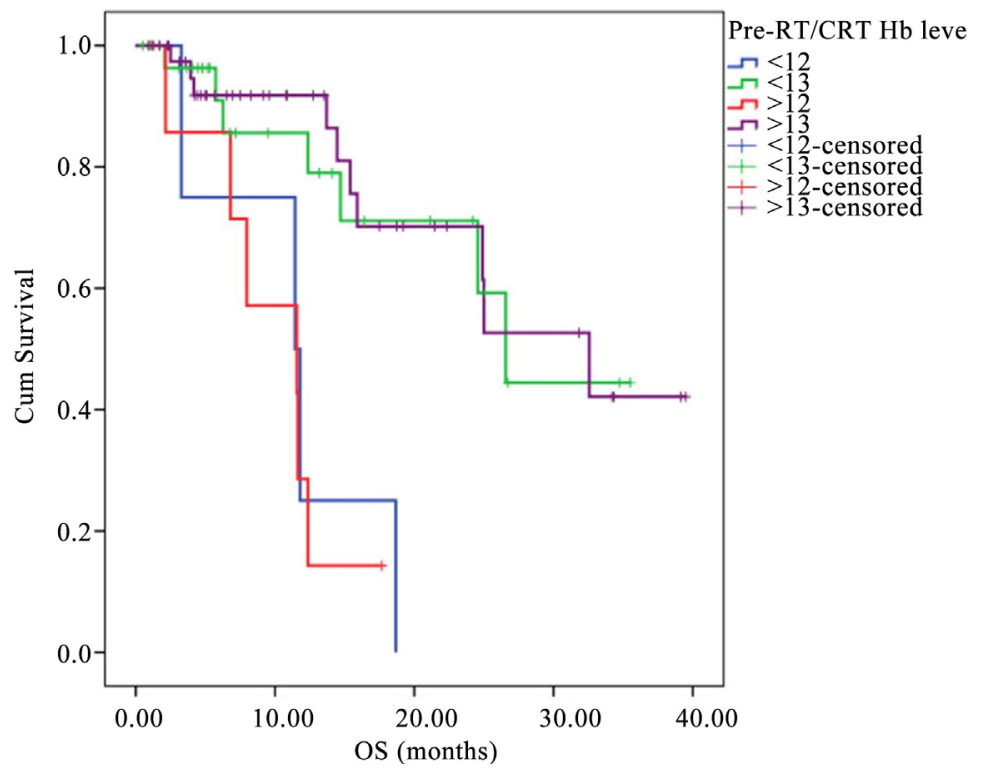

Figure 5. Effect of pre-RT/CRT hemoglobin level on OS $(n=86)$.

Patient, tumor and treatment characteristics in Pre-RT/CRT Hb $>12 \mathrm{~g} / \mathrm{dl}$ and $>13 \mathrm{~g} / \mathrm{dl}$ versus Pre-RT/CRT Hb<12 $\mathrm{g} / \mathrm{dl}$ and $<13 \mathrm{~g} / \mathrm{dl}$ (Table 6):

The total number of anemic patients was 35/86 (40.7\%) $(\mathrm{Hb}<12 \mathrm{~g} / \mathrm{dl}$ in $4 \mathrm{fe}-$ male patients and $\mathrm{Hb}<13 \mathrm{~g} / \mathrm{dl}$ in 31 male patients, $)(\mathrm{p}=0.000) .51 / 85$ patients were non-anemic $(\mathrm{Hb}>12 \mathrm{~g} / \mathrm{dl}$ in 7 female patients, and $\mathrm{Hb}>13 \mathrm{~g} / \mathrm{dl}$ in 43 male patients $(\mathrm{p}=0.000)$.

Anemic patients had predominance of PS 2 in 4/4 (100\%) of patients with $\mathrm{Hb}$ $<12 \mathrm{~g} / \mathrm{dl}$, versus PS 1 in 30/31 (96.8\%) of patients who had $\mathrm{Hb}<13 \mathrm{~g} / \mathrm{dl}$ ( $\mathrm{p}=$ 0.000). 6/8 (75\%) of patients with $\mathrm{Hb}>12 \mathrm{~g} / \mathrm{dl}$ had PS 2, compared to PS 1 in $41 / 43(95.3 \%)$ of patients with $\mathrm{HB}>13 \mathrm{~g} / \mathrm{dl}(\mathrm{p}=0.000)$. 
Table 6. Patient, tumor and treatment characteristics in anemic and non-anemic patients.

\begin{tabular}{|c|c|c|c|c|c|c|c|c|c|c|c|}
\hline & & \multicolumn{2}{|c|}{$<12(\mathrm{n}=4)$} & \multicolumn{2}{|c|}{$<13(\mathrm{n}=31)$} & \multirow{2}{*}{ P-value } & \multicolumn{2}{|c|}{$>12(n=8)$} & \multicolumn{2}{|c|}{$>13(n=43)$} & \multirow{2}{*}{ p-value } \\
\hline & & No. & $\%$ & No. & $\%$ & & No. & $\%$ & No. & $\%$ & \\
\hline \multirow{2}{*}{ Age } & $<56$ years & 2 & $50.0 \%$ & 11 & $35.5 \%$ & \multirow{2}{*}{0.762} & 4 & $50.0 \%$ & 24 & $55.8 \%$ & \multirow{2}{*}{0.572} \\
\hline & $>56$ years & 2 & $50.0 \%$ & 20 & $64.5 \%$ & & 4 & $50.0 \%$ & 19 & $44.2 \%$ & \\
\hline \multirow{3}{*}{$\begin{array}{l}\text { Performance } \\
\text { at presentation }\end{array}$} & 0 & 0 & $0.0 \%$ & 0 & $0.0 \%$ & \multirow{3}{*}{0.000} & 0 & $0.0 \%$ & 1 & $2.3 \%$ & \multirow{3}{*}{0.000} \\
\hline & 1 & 0 & $0.0 \%$ & 30 & $96.8 \%$ & & 2 & $25.0 \%$ & 41 & $95.3 \%$ & \\
\hline & 2 & 4 & $100.0 \%$ & 1 & $3.2 \%$ & & 6 & $75.0 \%$ & 1 & $2.3 \%$ & \\
\hline \multirow{2}{*}{ Gender } & Male & 0 & $0.0 \%$ & 31 & $100.0 \%$ & \multirow{2}{*}{0.000} & 1 & $12.5 \%$ & 43 & $100.0 \%$ & \multirow{2}{*}{0.000} \\
\hline & Female & 4 & $100.0 \%$ & 0 & $0.0 \%$ & & 7 & $87.5 \%$ & 0 & $0.0 \%$ & \\
\hline \multirow{2}{*}{ Site of the tumor } & Larynx & 0 & $0.0 \%$ & 31 & $100.0 \%$ & \multirow{2}{*}{0.000} & 3 & $37.5 \%$ & 43 & $100.0 \%$ & \multirow{2}{*}{0.000} \\
\hline & Hypopharynx & 4 & $100.0 \%$ & 0 & $0.0 \%$ & & 5 & $62.5 \%$ & 0 & $0.0 \%$ & \\
\hline \multirow[b]{2}{*}{ T-stage } & 1,2 & 0 & $0.0 \%$ & 12 & $38.7 \%$ & \multirow[b]{2}{*}{0.124} & 3 & $37.5 \%$ & 22 & $51.2 \%$ & \multirow[b]{2}{*}{0.477} \\
\hline & 3,4 & 4 & $100.0 \%$ & 19 & $61.3 \%$ & & 5 & $62.5 \%$ & 21 & $48.8 \%$ & \\
\hline \multirow{2}{*}{ N0 } & 0 & 2 & $50.0 \%$ & 18 & $58.1 \%$ & \multirow{2}{*}{0.759} & 1 & $12.5 \%$ & 30 & $69.8 \%$ & \multirow{2}{*}{0.002} \\
\hline & 1,2 & 2 & $50.0 \%$ & 13 & $41.9 \%$ & & 7 & $87.5 \%$ & 13 & $30.2 \%$ & \\
\hline \multirow{2}{*}{ Metastases } & M0 & 4 & $100.0 \%$ & 30 & $96.8 \%$ & & 7 & $87.5 \%$ & 42 & $97.7 \%$ & \\
\hline & M1 & 0 & $0.0 \%$ & 1 & $3.2 \%$ & 0.715 & 1 & $12.5 \%$ & 1 & $2.3 \%$ & 0.173 \\
\hline $\mathrm{St}_{\mathrm{S}}$ & 1,2 & 0 & $0.0 \%$ & 7 & $22.6 \%$ & م & 1 & $12.5 \%$ & 19 & $44.2 \%$ & 000 \\
\hline Jiage & 3,4 & 4 & $100.0 \%$ & 24 & $77.4 \%$ & & 7 & $87.5 \%$ & 24 & $55.8 \%$ & \\
\hline & Yes & 0 & $0.0 \%$ & 15 & $48.4 \%$ & & 2 & $25.0 \%$ & 19 & $44.2 \%$ & \\
\hline Surgery & No & 4 & $100.0 \%$ & 13 & $41.9 \%$ & 0.362 & 6 & $75.0 \%$ & 21 & $48.8 \%$ & 0.092 \\
\hline & NA & 0 & $0.0 \%$ & 3 & $9.7 \%$ & & 0 & $0.0 \%$ & 3 & $7.0 \%$ & \\
\hline & Yes & 2 & $50.0 \%$ & 3 & $9.7 \%$ & & 5 & $62.5 \%$ & 3 & $7.0 \%$ & \\
\hline $\begin{array}{c}\text { Induction } \\
\text { chemotherapy }\end{array}$ & No & 1 & $25.0 \%$ & 25 & $80.6 \%$ & 0.000 & 3 & $37.5 \%$ & 36 & $83.7 \%$ & 0.044 \\
\hline & NA & 1 & $25.0 \%$ & 3 & $9.7 \%$ & & 0 & $0.0 \%$ & 4 & $9.3 \%$ & \\
\hline & Yes & 3 & $75.0 \%$ & 21 & $67.7 \%$ & & 4 & $50.0 \%$ & 31 & $72.1 \%$ & \\
\hline $\begin{array}{c}\text { Radiation } \\
\text { (alone or concurrent) }\end{array}$ & No & 0 & $0.0 \%$ & 7 & $22.6 \%$ & 0.132 & 4 & $50.0 \%$ & 8 & $18.6 \%$ & 0.436 \\
\hline & NA & 1 & $25.0 \%$ & 3 & $9.7 \%$ & & 0 & $0.0 \%$ & 4 & $9.3 \%$ & \\
\hline גד & $<66$ & 3 & $100.0 \%$ & 20 & $83.3 \%$ & -5 & 1 & $25.0 \%$ & 21 & $61.8 \%$ & 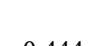 \\
\hline 121 cose & $>66$ & 0 & $0.0 \%$ & 4 & $16.7 \%$ & 0.13 & 3 & $75.0 \%$ & 13 & $38.2 \%$ & 0.774 \\
\hline & Yes & 2 & $50.0 \%$ & 10 & $32.3 \%$ & & 2 & $25.0 \%$ & 17 & $39.5 \%$ & \\
\hline $\begin{array}{c}\text { Concurrent } \\
\text { chemoradiation CCRT }\end{array}$ & No & 1 & $25.0 \%$ & 18 & $58.1 \%$ & 0.402 & 6 & $75.0 \%$ & 22 & $51.2 \%$ & 0.413 \\
\hline & NA & 1 & $25.0 \%$ & 3 & $9.7 \%$ & & 0 & $0.0 \%$ & 4 & $9.3 \%$ & \\
\hline & No & 0 & $0.0 \%$ & 4 & $12.9 \%$ & & 4 & $50.0 \%$ & 9 & $20.9 \%$ & \\
\hline Acute RT & NA & 1 & $25.0 \%$ & 3 & $9.7 \%$ & $1-$ & 0 & $0.0 \%$ & 3 & $7.0 \%$ & \\
\hline toxicity & Mucositis & 3 & $75.0 \%$ & 21 & $67.7 \%$ & 0.150 & 2 & $25.0 \%$ & 26 & $60.5 \%$ & 0.040 \\
\hline & Skin reaction & 0 & $0.0 \%$ & 3 & $9.7 \%$ & & 2 & $25.0 \%$ & 5 & $11.6 \%$ & \\
\hline
\end{tabular}




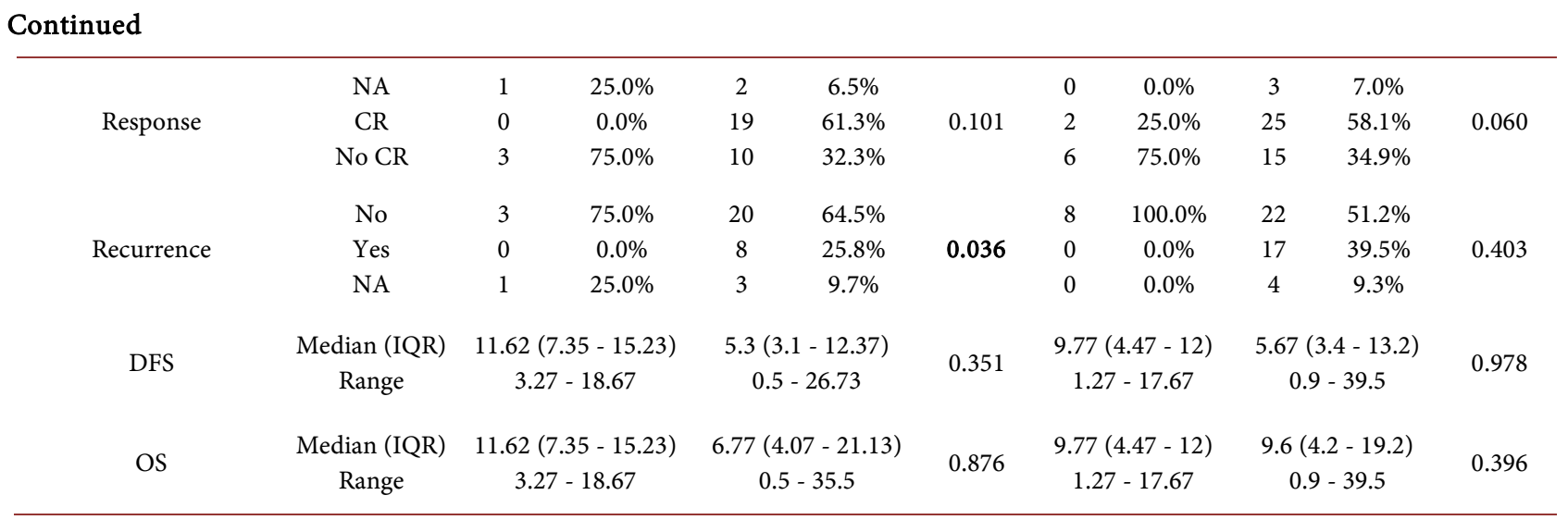

4/4 (100\%) of patients with $\mathrm{Hb}<12 \mathrm{~g} / \mathrm{dl}$ had hypopharyngeal SCC and 31/31 $(100 \%)$ of patients with $<13 \mathrm{~g} / \mathrm{dl}$ had laryngeal SCC $(\mathrm{p}=0.000)$. Whereas, 5/8 (62.5\%) of patients with $\mathrm{Hb}>12$ had hypopharyngeal SCC versus 43/43 (100\%) of patients with $\mathrm{Hb}>13 \mathrm{~g} / \mathrm{dl}$ who had laryngeal SCC $(\mathrm{p}=0.000)$.

Node positive disease occurred in 4/4 (100\%) of patients who had $\mathrm{Hb}<12$ $\mathrm{g} / \mathrm{dl}$ and in $18 / 31$ (58.1\%) of patients who had $\mathrm{Hb}<13 \mathrm{~g} / \mathrm{dl}$ ( $\mathrm{p} 0.759)$. While, $7 / 8$ (87.5\%) of patients with $\mathrm{Hb}>12 \mathrm{~g} / \mathrm{dl}$ and $13 / 34$ (30.2\%) of patients with $\mathrm{Hb}>13$ $\mathrm{g} / \mathrm{dl}$ had nodal positive disease $(\mathrm{p}=0.002)$. Disease stage 3,4 was diagnosed in $4 / 4(100 \%)$ of patients with $\mathrm{Hb}<12 \mathrm{~g} / \mathrm{dl}$ and in $24 / 31(77.4 \%)$ of patients who had $\mathrm{Hb}<13 \mathrm{~g} / \mathrm{dl}$ (p 0.092). On the other hand, 7/8 (87.5\%) of patients who had $\mathrm{Hb}>12 \mathrm{~g} / \mathrm{dl}$ and 24/43 (55.8\%) of patients who had $\mathrm{Hb}>13 \mathrm{~g} / \mathrm{dl}$ were diagnosed with advanced stage disease $(\mathrm{p}=0.288)$.

More patients with $\mathrm{Hb}<12 \mathrm{~g} / \mathrm{dl} 2 / 4(50 \%)$ received induction chemotherapy than patients with $\mathrm{Hb}<13 \mathrm{~g} / \mathrm{dl} 3 / 31(9.7 \%)$, ( $\mathrm{p}=0.000)$. Also more patients with $\mathrm{Hb}>12 \mathrm{~g} / \mathrm{dl} 5 / 8(62.5 \%)$ had induction chemotherapy than those with $\mathrm{Hb}>13$ $\mathrm{g} / \mathrm{dl} 3 / 43(7 \%),(\mathrm{p}=0.044)$. Concurrent chemoradiotherapy (adjuvant or definitive) was administered in $2 / 4(50 \%)$ and in $10 / 31$ (32.3\%) of patients with $\mathrm{Hb}<$ 12 and $<13 \mathrm{~g} / \mathrm{dl}(\mathrm{p}=0.402)$ respectively, whereas CRT was given in $2 / 8(25 \%)$ and $17 / 43(39.5 \%)$ of patients with pretreatment $\mathrm{Hb}>12$ and $>13 \mathrm{~g} / \mathrm{dl}$ respectively $(\mathrm{p}=0.413)$. Most of the anemic patients received RT ( $50 \%$ of patients with $\mathrm{Hb}<12 \mathrm{~g} / \mathrm{dl}$, and $67 \%$ of cases with $\mathrm{Hb}<13 \mathrm{~g} / \mathrm{dl}, \mathrm{p}=0.132)$. Similarly, patients with $\mathrm{Hb}>12$ and $>13 \mathrm{~g} / \mathrm{dl}$ were treated with $\mathrm{RT}$ ( $50 \%$ and $72.1 \%$ respectively, $\mathrm{p}$ $=0.436)$. Although non-significant acute mucositis tended to occur more in anemic patients $(75.0 \%$ of patients with $\mathrm{Hb}<12 \mathrm{~g} / \mathrm{dl}$, and $61.7 \%$ of patients with $\mathrm{Hb}<13 \mathrm{~g} / \mathrm{dl}, \mathrm{p}=0.156$ ), versus acute mucositis in $25 \%$ of patients with $\mathrm{Hb}>12$ $\mathrm{g} / \mathrm{dl}$ and $60.5 \%$ of patients who had $\mathrm{Hb}>13 \mathrm{~g} / \mathrm{dl}(\mathrm{p}=0.646)$.

Comparing response to treatment, $0 / 4$ of patients with $\mathrm{Hb}<12 \mathrm{~g} / \mathrm{dl}$ had CR, while 19/31 (61.3\%) of $\mathrm{Hb}<13 \mathrm{~g} / \mathrm{dl}$ patients achieved CR $(\mathrm{p}=0.101)$. The incidence of CR was $2 / 8$ (25.0\%) of patients who had $\mathrm{Hb}>12 \mathrm{~g} / \mathrm{dl}$ versus $25 / 31$ (58.1\%) CR achieved in patients with $\mathrm{Hb}>13 \mathrm{~g} / \mathrm{dl}(\mathrm{p}=0.060)$. No recurrence developed in anemic patients with $\mathrm{Hb}<12(0 / 4)$, however 8/31 (25.8\%) of anemic patients with $\mathrm{Hb}<13 \mathrm{~g} / \mathrm{dl}(\mathrm{p}=0.036)$ had local recurrence. As regards 
the non-anemic group, patients who had $\mathrm{Hb}>12 \mathrm{~g} / \mathrm{dl}(0 / 8)$ developed no recurrence but it occurred in 17/43 (39\%) of patients with $\mathrm{Hb}>13 \mathrm{~g} / \mathrm{dl}(\mathrm{p}=0.403)$.

Table 7 demonstrates that non-anemic female patients had relatively longer mean DFS than non-anemic male patients (7.97 and 6.30 months respectively, $p$ = 0.127); anemic females also achieved longer mean DFS compared to anemic male patients (11.62 and 5.30 months respectively, $\mathrm{p}=0.301)$. In general males (anemic and non-anemic) had better mean OS than females (anemic and nonanemic). Where, males with $\mathrm{Hb}>13 \mathrm{~g} / \mathrm{dl}$ survived for 32.567 months and females who had $\mathrm{Hb}>12 \mathrm{~g} / \mathrm{dl}$ survived for 7.967 months $(\mathrm{p}=0.001)$. Similarly males with $\mathrm{Hb}<13 \mathrm{~g} / \mathrm{dl}$ compared to females with $\mathrm{Hb}<12 \mathrm{~g} / \mathrm{dl}$ had better mean OS (26.567 months and 11.433 months respectively, $\mathrm{p}=0.004)$ (Table 8 , Figure 6 and Figure 7).

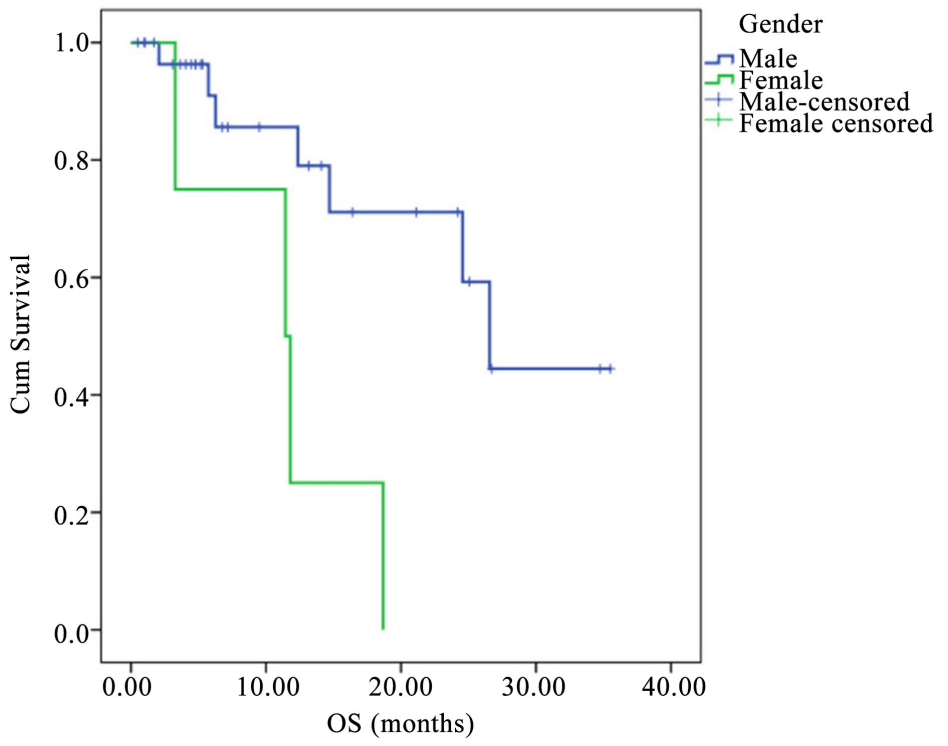

Figure 6. Effect of gender on OS in anemic patients $(\mathrm{n}=35)$.

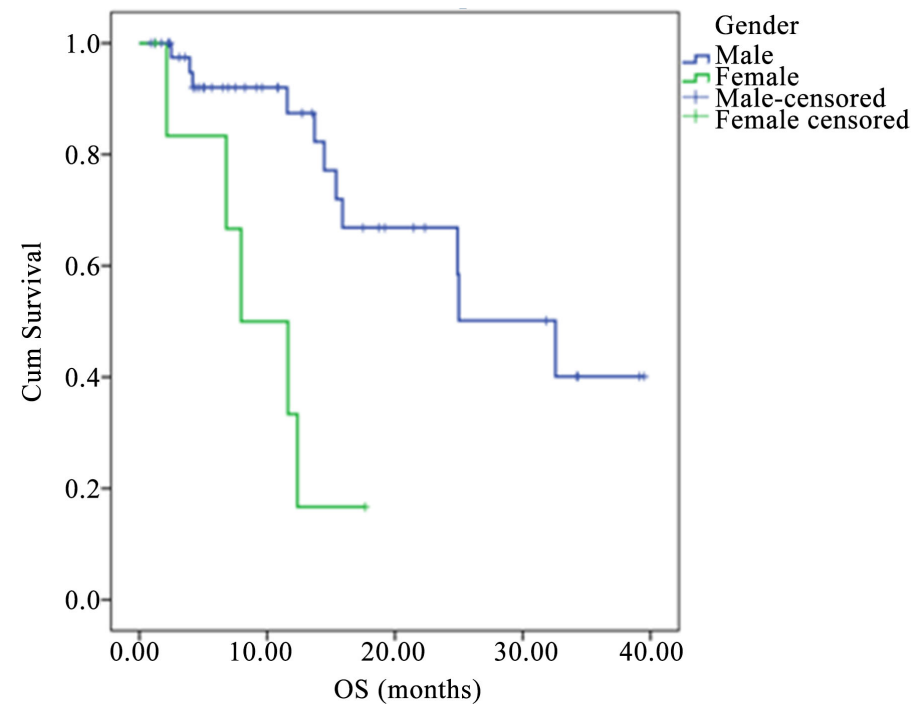

Figure 7. Effect of gender on OS in non-anemic patients $(n=51)$. 
Table 7. Comparison of DFS between anemic and non-anemic patients.

\begin{tabular}{cccccccc}
\hline & & \multicolumn{2}{c}{ DFS } & \multicolumn{2}{c}{$95 \% \mathrm{CI}$} & \multicolumn{2}{c}{ Log rank test } \\
\cline { 3 - 7 } & No. & Mean & SE & Lower & Upper & $\mathrm{X}^{2}$ & P-value \\
\hline No anemia & & & & & & & \\
Male $\mathrm{Hb}>13$ & 44 & 6.30 & 10.39 & 3.48 & 12.97 & 2.331 & 0.127 \\
Female $\mathrm{Hb}>12$ & 7 & 7.97 & 5.84 & 2.13 & 12.37 & & \\
Anemia & & & & & & & \\
Male $\mathrm{Hb}<13$ & 31 & 5.30 & 6.89 & 3.10 & 12.37 & 1.070 & 0.301 \\
Female $\mathrm{Hb}<12$ & 4 & 11.62 & 6.30 & 7.35 & 15.23 & & \\
Overall & 86 & 20.200 & 2.314 & 15.664 & 24.736 & & \\
\hline
\end{tabular}

Table 8. Comparison of OS in anemic and non-anemic patients.

\begin{tabular}{cccccccc}
\hline & No. & \multicolumn{3}{c}{ OS } & \multicolumn{2}{c}{$95 \% \mathrm{CI}$} & \multicolumn{2}{c}{ Log rank test } \\
\cline { 3 - 7 } & & Mean & SE & Lower & Upper & $\mathrm{X}^{2}$ & p-value \\
\hline No anemia & & & & & & & \\
Male $\mathrm{Hb}>13$ & 44 & 32.567 & 5.665 & 21.463 & 43.671 & 10.648 & 0.001 \\
Female $\mathrm{Hb}>12$ & 7 & 7.967 & 2.960 & 2.165 & 13.768 & & \\
Anemia & & & & & & & \\
Male $\mathrm{Hb}<13$ & 31 & 26.567 & 2.265 & 22.126 & 31.007 & 8.125 & 0.004 \\
Female $\mathrm{Hb}<12$ & 4 & 11.433 & 4.267 & 3.071 & 19.796 & & \\
Overall & 86 & 24.900 & 4.301 & 16.469 & 33.331 & & \\
\hline
\end{tabular}

\section{Discussion}

Regardless of the etiology of anemia, its existence in cancer patients can impair treatment effectiveness and outcome and also negatively impact the quality of life of cancer patients [8]. Nutritional anemia, namely iron deficiency anemia (ID), has three- to four-times higher prevalence in the developing world than the developed world especially in population with more physiologic needs [34].

The incidence of ID anemia increases after 50 years of age [35]. The median age of patients in our study was 56 years which is the same age reported by Narayanaswamy RK et al. [13] in their analysis of pre-CRT anemia in 91 patients with HNSCC. Anemia was diagnosed in $36.4 \%$ of our female patients and $41.3 \%$ of our male patients. In contrast, Dubray et al. [7] who defined anemia as hemoglobin level $<13.5 \mathrm{~g} / \mathrm{dl}$ in men and $<12.0 \mathrm{~g} / \mathrm{dl}$ in women, diagnosed anemia in $31 \%$ of men and $17 \%$ of women.

The incidence of anemia varies by tumor site. Dubray et al. [7] reported that the incidence of pre-RT anemia was $40 \%$ (20 of 50) for supraglottic SCC versus $12 \%$ (25 of 205) for glottic SCC. In the current study the incidence of pre-RT/CRT $\mathrm{Hb}<12 \mathrm{~g} / \mathrm{dl}$ was 100\% in hypopharyngeal SCC and pre-RT/CRT $\mathrm{Hb}<13 \mathrm{~g} / \mathrm{dl}$ had $100 \%$ incidence in laryngeal SCC.

Pretreatment anemia was significantly associated with higher T- or $\mathrm{N}$-stage in 
patients with HNSCC studied by Dubray et al. [7]. Blitzer et al. [36] reported that low $\mathrm{Hb}$ levels were associated with advanced T-stage HNSCC treated with definitive radiation. We also observed that patients diagnosed with T3 - 4 tumor tended to have low pretreatment $\mathrm{Hb}$ levels $<12$ and $<13 \mathrm{~g} / \mathrm{dl}$ (100\% and $61.3 \%$ respectively, $\mathrm{p}=0.124)$. Advanced nodal disease was detected in $50 \%$ of patients with $\mathrm{Hb}$ levels $<12 \mathrm{~g} / \mathrm{dl}$, whereas $58.1 \%$ of cases with $\mathrm{Hb}<13 \mathrm{~g} / \mathrm{dl}$ were node negative $(\mathrm{p}=0.759)$. Node positive disease was diagnosed in $87.5 \%$ of patients with $\mathrm{Hb}>12 \mathrm{~g} / \mathrm{dl}$, while $69.8 \%$ of $\mathrm{Hb}$ level $>13 \mathrm{~g} / \mathrm{dl}$ was detected in patients with node negative tumors $(\mathrm{p}=0.002)$.

With the increased use of concurrent chemotherapy with definitive radiation in HNSCC, most patients developed anemia [37] [38]. The incidence of pre-CRT $\mathrm{Hb}<12 \mathrm{~g} / \mathrm{dl}$ was $67 \%$, while pre-CRT $\mathrm{Hb} \geq 12 \mathrm{~g} / \mathrm{dl}$ was $24 \%$ in 91 Indian patients with different sites head and neck SCC [13]. In the current study we found a higher incidence of pre-RT/CRT $\mathrm{Hb}<12 \mathrm{~g} / \mathrm{dl}$ in $50 \%$ of patients who received CRT, compared to $32.3 \%$ incidence in patients with $\mathrm{Hb}<13 \mathrm{~g} / \mathrm{dl}$ treated with CRT ( $\mathrm{p}=0.402$ ). On the other hand only $25 \%$ and $39.5 \%$ of patients who were given CRT had Hb levels $>12$ and $>13 \mathrm{~g} / \mathrm{dl}$ respectively $(\mathrm{p}=0.413) .75 \%$ of our patients who were treated with induction chemotherapy also had a high incidence of $\mathrm{Hb}<12 \mathrm{~g} / \mathrm{dl}$, but much less incidence in patients who had pretreatment $\mathrm{Hb}<13 \mathrm{~g} / \mathrm{dl}(\mathrm{p}=0.000)$.

Narayanaswamy et al. [13] compared toxicity of CRT below and above $\mathrm{Hb}$ $10.7 \mathrm{~g} / \mathrm{dl}$ which is the common mean $\mathrm{Hb}$ level in India. The authors found that patients with pre-RT $\mathrm{Hb} \geq 10.7 \mathrm{~g} / \mathrm{dl}$ had lesser grade of mucositis compared to patients with pre-RT $\mathrm{Hb}<10.7 \mathrm{~g} / \mathrm{dl}$. Mucositis reaction associated with pre-RT $\mathrm{Hb}$ level showed statistical significance $(\mathrm{p}<0.0001)$. In agreement, our anemic cohort developed acute mucositis during radiotherapy (alone or combined with chemotherapy) in $75 \%$ of patients with pretreatment $\mathrm{Hb}<12 \mathrm{~g} / \mathrm{dl}$ and $67.75 \%$ of patients with $\mathrm{Hb}<13 \mathrm{~g} / \mathrm{dl}(\mathrm{p}=0.156)$. Acute mucositis tended to occur less with pre-RT/CRT Hb levels $>12$ and $>13 \mathrm{~g} / \mathrm{dl}(\mathrm{p}=0.646)$.

The occurrence of anemia also varies by timing of measurements, where $\mathrm{Du}$ bray et al. [7], showed that the incidence of pretreatment anemia was 40\% (20 of 50 ) for supraglottic laryngeal SCC versus $12 \%$ (25 of 205) for glottic SCC. The incidence of post-RT anemia was 56\% (25 of 45) for supraglottic SCC versus $23 \%$ (41 of 175) for glottic SCC. Welsh et al. [39], prospectively studied 20 patients with HNSCC, the pre-CRT Hb was in the normal range but dropped to < $11.5 \mathrm{~g} / \mathrm{dl}$ in $15 \%$ and $>11.5 \mathrm{~g} / \mathrm{dl}$ in $85 \%$ of patients after treatment. Chua et al. [11], randomized 334 patients with advanced nasopharyngeal SCC into RT alone arm and induction chemotherapy followed by RT arm. In the chemotherapy arm, the mean baseline, pre-radiation, and mid-radiation $\mathrm{Hb}$ levels were 13.6, 11.0 , and $11.8 \mathrm{~g} / \mathrm{dL}$, respectively. In the radiotherapy arm, the mean baseline pre-radiation and mid-radiation $\mathrm{Hb}$ levels were 13.7 and $12.9 \mathrm{~g} / \mathrm{dL}$, respectively. Multivariate analysis showed that a low mid-radiation $\mathrm{Hb}$ level, but not a low baseline or pre-radiation $\mathrm{Hb}$ level, was an independent predictor of local disease recurrence and malignancy-related death. We did not assess post-treatment $\mathrm{Hb}$ 
level in this report.

Our analysis of prognostic factors influencing treatment response noted that gender, site of the tumor, $\mathrm{T}$-stage, $\mathrm{N}$-stage and pre-RT/CRT Hb level were significant factors. Significant factors of overall survival included age, gender, performance status, $\mathrm{N}$-stage and pretreatment $\mathrm{Hb}$. No factors influenced the DFS ( $\mathrm{p}>0.05)$. Narayanaswamy et al. [13], in their analysis of 91 patients with HNSCC for the effect of pre-RT Hb levels in locally advanced HNSCC treated with CCRT, the authors found that performance status, pre-RT hemoglobin level, radiotherapy interruptions $>5$ days and non-development of grade III mucositis were significantly associated with good locoregional control. Pre-RT Hb in HNSCC was shown by many studies to be an independent significant prognostic factor of response and survival [10] [36] [40]. Lower levels of pretreatment $\mathrm{Hb}$ were significantly associated with reduced local control and response at the tumor primary site [7] [9] [36]. Warde et al. [41] studied pre-RT anemia in 735 patients with T1-2N0 glottic SCC. Patients diagnosed with a pretreatment $\mathrm{Hb}<$ $12 \mathrm{~g} / \mathrm{dL}$ were found through multivariate analysis to have a 1.8 -fold increased risk for locoregional failure ( $95 \%$ confidence interval, 1.2 - 2.5) compared to patients with $\mathrm{Hb} 15.0 \mathrm{~g} / \mathrm{dL}$. Similarly, we demonstrated that pretreatment $\mathrm{Hb}$ was among the significant prognostic factors predicting response to treatment, where $58.7 \%$ of $\mathrm{Hb}$ levels $>12 \mathrm{~g} / \mathrm{dl}$ and $>13 \mathrm{~g} / \mathrm{dl}$ were associated with CR compared to $41.3 \%$ of $\mathrm{Hb}$ levels $<12$ and $13 \mathrm{~g} / \mathrm{dl}(\mathrm{p}=0.034)$. Narayanaswamy RK et al. [13] detected significantly better response (CR versus no $\mathrm{CR}$ ) to treatment in patients with pre-RT $\mathrm{Hb} \geq 12 \mathrm{~g} / \mathrm{dl}$ than $\mathrm{Hb}<12 \mathrm{~g} / \mathrm{dl}$ ( $\mathrm{p}<0.001$ ). Blitzer et al. [36] demonstrated that low $\mathrm{Hb}$ levels were associated with local recurrence in advanced T-stage HNSCC patients treated with definitive radiation. The authors predicted an approximately $10 \%$ drop in locoregional control for every 2-g/dL drop (estimated 2-year locoregional control $26 \%$ for $\mathrm{Hb}=12 \mathrm{~g} / \mathrm{dL}, 37 \%$ for $\mathrm{Hb}=14 \mathrm{~g} / \mathrm{dL}$, and $47 \%$ for $\mathrm{Hb}=16)$. Few studies showed no correlation between local tumor control and pretreatment $\mathrm{Hb}$ in glottic SCC [42] [43] [44]. In a multivariate analysis of 116 patients with advanced unresectable HNSCC treated with CRT Haddad et al. [9] reported that pre-CRT $\mathrm{Hb}<12 \mathrm{~g} / \mathrm{dl}$ was associated with a worse response rate at the primary site $(\mathrm{p}=0.05)$.

Pre-treatment $\mathrm{Hb}$ levels have been shown to be associated with overall and disease-free survival in HNSCC among other solid tumors [8] [15]. This correlation of pre-treatment anemia and outcome is documented with radiation therapy and surgery in HNSCC patients [45]. The majority of studies found that a low pre-treatment $\mathrm{Hb}$ level is a significant prognostic factor of local control and survival after curative radiotherapy independent of tumor factors as T-stage and $\mathrm{N}$-stage. Low Hb levels were associated with worse survival [42] [43]. The levels of pre-treatment $\mathrm{Hb}$ in head and neck carcinomas that corresponded with poor OS and DFS outcome have been defined by retrospective studies to be $9,12,13$, and $14.5 \mathrm{~g} / \mathrm{dl}$ [7] [8] [10] [46]. According to Vaupel $\mathrm{P}$ et al. [23] the range of $\mathrm{Hb}$ levels considered optimal for tumor oxygenation was $12-14 \mathrm{~g} / \mathrm{dl}$. It is likely that the $\mathrm{Hb}$ threshold depends on the specific type of malignancy, the timing of $\mathrm{Hb}$ 
measurement, and addition of chemotherapy [11]. In accordance we identified pre-RT/CRT Hb level as one of the significant prognostic factors determining incidence of OS, where $\mathrm{Hb}$ levels $<12$ and $13 \mathrm{~g} / \mathrm{dl}$ versus $\mathrm{Hb}$ levels $>12$ and $>13$ $\mathrm{g} / \mathrm{dl}$ were associated with 21.3 and 27.2 months median OS $(\mathrm{p}=0.000)$. However, the pretreatment $\mathrm{Hb}$ levels did not affect the incidence of median DFS of anemic and non-anemic patients $(19.442,19.496$ months respectively, $\mathrm{p}=0.23)$. Haddad et al. [9] did not observe a statistically significant impact of pretreatment $\mathrm{Hb}$ level on survival of HNSCC patients, where patients with $\mathrm{Hb}<12 \mathrm{~g} / \mathrm{dl}$ had a relatively better DFS $(\mathrm{p}=0.109)$, while patients with $\mathrm{Hb}>12 \mathrm{~g} / \mathrm{dl}$ had better OS $(\mathrm{p}=0.656)$.

The shortcomings of our study include the retrospective nature of the study, and the small number of patients in comparison to many similar studies in the literature. However, to the best of our knowledge this is the first study in Egypt of the frequency of anemia in head and neck SCC patients before radiotherapy and/or chemoradiotherapy and its effect on response to treatment and survival.

\section{Conclusion}

Anemia is a frequent problem in patients with head and neck squamous cell carcinoma. Evidence from the literature emphasizes the independent prognostic role of anemia for recurrence and survival which calls for formal protocol for hemoglobin monitoring before, during and after treatment. The Hb threshold which affects treatment outcome needs to be defined where it may vary according to the tumor site, from patient to patient, and timing of measurement. The hematologic definition of anemia may not necessarily be the oncologic definition [12]. In Egypt nutritional iron deficiency anemia is prevalent and is a common laboratory finding in head and neck carcinoma patients presenting to our institution.

\section{References}

[1] Jemal, A., Siegel, R., Xu, J. and Ward, E. (2010) Cancer Statistics, 2010. CA: A Cancer Journal for Clinicians, 60, 277-300. https://doi.org/10.3322/caac.20073

[2] Casciato, D.A. and Territo, M.C. (2009) Manual of Clinical Oncology. 6th Edition, Lippincott Williams \& Wilkins, Philadelphia, 169-188.

[3] Adelstein, D.J., Li, Y., Adams, G.L., Wagner Jr., H., Kish, J.A., Ensley, J.F., et al. (2003) An Intergroup Phase III Comparison of Standard Radiation Therapy and Two Schedules of Concurrent Chemoradiotherapy in Patients with Unresectable Squamous Cell Head and Neck Cancer. Journal of Clinical Oncology, 21, 92-98. https://doi.org/10.1200/JCO.2003.01.008

[4] Ludwig, H., Van Belle, S., Barrett-Lee, P., et al. (2004) The European Cancer Anaemia Survey (ECAS): A Large, Multinational, Prospective Survey Defining the Prevalence, Incidence, and Treatment of Anemia in Cancer Patients. European Journal of Cancer, 40, 2293-2306. https://doi.org/10.1016/j.ejca.2004.06.019

[5] Caro, J.J., Salas, M., Ward, A. and Goss, G. (2001) Anemia as an Independent Prognostic Factor for Survival in Patients with Cancer: A Systemic, Quantitative Review. Cancer, 91, 2214-2221. 
[6] Fein, D.A., Lee, W.R., Hanlon, A.L., et al. (1995) Pretreatment Hemoglobin Level Influences Local Control and Survival of T1-T2 Squamous Cell Carcinomas of the Glottic Larynx. Journal of Clinical Oncology, 13, 2077-2083. https://doi.org/10.1200/JCO.1995.13.8.2077

[7] Dubray, B., Mosseri, V., Brunin, F., et al. (1996) Anemia Is Associated with Lower Local-Regional Control and Survival after Radiation Therapy for Head and Neck Cancer: A Prospective Study. Radiology, 201, 553-558. https://doi.org/10.1148/radiology.201.2.8888257

[8] Kumar, P. (2000) Impact of Anaemia in Patients with Head and Neck Cancer. The Oncologist, 5, 13-18. https://doi.org/10.1634/theoncologist.5-suppl_2-13

[9] Haddad, R., Suntharalingam, M., Chen, T., et al. (2001) Pretreatment Hemoglobin Is Associated with Response to Chemoradiation Therapy (CRT) in Patients with Advanced Unresectable Squamous Cell Carcinoma of the Head and Neck. Proceedings of the American Society of Clinical Oncology, 19, 417a.

[10] Schafer, U., Micke, O., Muller, S.B., Schuller, P. and Willich, N. (2003) Hemoglobin as an Independent Prognostic Factor in the Radiotherapy of Head and Neck Tumors. Strahlentherapie und Onkologie, 179, 527-534.

https://doi.org/10.1007/s00066-003-1117-x

[11] Chua, D.T., Sham, J.S. and Choy, D.T. (2004) Prognostic Impact of Hemoglobin Levels on Treatment Outcome in Patients with Nasopharyngeal Carcinoma Treated with Sequential Chemoradiotherapy or Radiotherapy Alone. Cancer, 101, 307-316. https://doi.org/10.1002/cncr.20366

[12] Hu, K. and Harrison, L.B. (2005) Impact of Anemia in Patients with Head and Neck Cancer Treated with Radiation Therapy. Current Treatment Options in Oncology, 6, 31-45. https://doi.org/10.1007/s11864-005-0011-4

[13] Narayanaswamy, R.K., Potharaju, M., Vaidhyswaran, A.N. and Perumal, K. (2015) Pre-Radiotherapy Haemoglobin Level Is A Prognosticator in Locally Advanced Head and Neck Cancers Treated with Concurrent Chemoradiation. Journal of Clinical and Diagnostic Research, 9, XC14-XC18. https://doi.org/10.7860/JCDR/2015/11593.6102

[14] Stasi, R., Abriani, L., Beccaglia, P., et al. (2003) Cancer-Related Fatigue: Evolving Concepts in Evaluation and Treatment. Cancer, 98, 1786-1801. https://doi.org/10.1002/cncr.11742

[15] Harrison, L.B., Shasha, D. and Homel, P. (2002) Prevalence of Anemia in Cancer Patients Undergoing Radiotherapy: Prognostic Significance and Treatment. Oncology, 63, 11-18. https://doi.org/10.1159/000067147

[16] Hu, K., Sachoeva, G., Culliney, B., et al. (2003) Accelerated Fractionated Radiation by Concomitant Boost (AFX-CB) with Concurrent cis-Platinum for Advanced Nasopharyngeal Cancer. Paper presented at the 85 th Annual Meeting of the American Radium Society, Chicago, IL.

[17] Tarnawski, R., Skladowski, K. and Maciejewski, B. (1997) Prognostic Value of Hemoglobin Concentration in Radiotherapy for Cancer of Supraglottic Larynx. International Journal of Radiation Oncology ${ }^{\star}$ Biology ${ }^{\star}$ Physics, 38, 1007-1011. https://doi.org/10.1016/S0360-3016(97)00308-8

[18] Skladowski, K., Tarnawski, R., Maciejewski, B., et al. (1999) Clinical Radiobiology of Glottic T1 Squamous Cell Carcinoma. International Journal of Radiation Oncology ${ }^{\star}$ Biology ${ }^{\star}$ Physics, 43, 101-106. https://doi.org/10.1016/S0360-3016(98)00375-7

[19] van de Pol, S.M., Doornaert, P.A., de Bree, R., Leemans, C.R., Slotman, B.J. and Langendijk, J.A. (2006) The Significance of Anemia in Squamous Cell Head and 
Neck Cancer Treated with Surgery and Postoperative Radiotherapy. Oral Oncology, 42, 131-138. https://doi.org/10.1016/j.oraloncology.2005.06.021

[20] Huang, M., Chen, Q., Xiao, J., Yao, T., Bian, L., Liu, C. and Lin, Z. (2014) Overexpression of Hypoxia-Inducible Factor-1Alpha Is a Predictor of Poor Prognosis in Cervical Cancer: A Clinicopathologic Study and a Meta-Analysis. International Journal of Gynecological Cancer, 24, 1054-1064. https://doi.org/10.1097/IGC.0000000000000162

[21] Littlewood, T.J. (2001) The Impact of Hemoglobin Levels on Treatment Outcomes in Patients with Cancer. Seminars in Oncology, 28, 49-53. https://doi.org/10.1016/S0093-7754(01)90213-1

[22] Dietl, B., Marienhagen, J., Schafer, C. and Kolbl, O. (2007) The Prognostic Value of Anemia at Different Treatment Times in Patients with Locally Advanced Head and Neck Cancer Treated with Surgery and Postoperative Radiotherapy. Clinical Oncology, 19, 228-233. https://doi.org/10.1016/j.clon.2007.02.009

[23] Vaupel, P., Thews, O., Mayer, A., Höckel, S. and Höckel, M. (2002) Oxygenation Status of Gynecologic Tumours: What Is the Optimal Haemoglobin Level? Strahlentherapie und Onkologie, 178, 727-731.

[24] Klap, P.C. and Hemphill, R.R. (2011) Acquired Hemolytic Anemia. In: Tintinalli, J.E., Stapczynski, J.S., Cline, D.M., Ma, O.J., Cydulka, R.K. and Meckler, G.D., Eds., Tintinallis Emergency Medicine: A Comprehensive Study Guide, 7th Edition, McGraw-Hill, New York.

[25] Park, S., Jung, C.W., Kim, K., Kim, S.J., Kim, S.W. and Jang, J.H. (2015) Iron Deficient Erythropoiesis Might Play Key Role in Development of Anemia in Cancer Patients. Oncotarget, 6, 42803-42812. https://doi.org/10.18632/oncotarget.5658

[26] Grotto, H.Z. (2008) Anaemia of Cancer: An Overview of Mechanisms Involved in Its Pathogenesis. Medical Oncology, 25, 12-21.

[27] Ludwig, H., Muldur, E., Endler, G. and Hubl, W. (2013) Prevalence of Iron Deficiency across Different Tumors and Its Association with Poor Performance Status, Disease Status and Anemia. Annals of Oncology, 24, 1886-1892.

[28] Winter, S.C.A., Corbridge, R.J., Cox, G.J. and Harris, A.L. (2005) Hypoxia and Anemia in Head and Neck Squamous Cell Carcinoma-Mechanisms of Therapy Failure and Provision of New Therapeutic Targets. Clinical Otolaryngology, 30, 99-104.

[29] Surono, A., Priyanto, P. and Indrasari, S.R. (2016) Hypoxia-Inducible Factor-1 $\alpha$ Expression in Indonesian Laryngeal Squamous Cell Carcinoma Patients. Journal of Oncology, 2016, Article ID: 3215463. https://doi.org/10.1155/2016/3215463

[30] WHO (2011) Hemoglobin Concentrations for the Diagnosis of Anemia and Assessment of Severity. Vitamin and Mineral Nutrition Information System. WHO/NMH/NHD/MNM/11.1, World Health Organization, Geneva.

[31] Eisenhauer, E.A., Therasse, P., Bogaerts, J., Schwartz, L.H., Sargent, D., Ford, R., et al. (2009) New Response Evaluation Criteria in Solid Tumours: Revised RECIST Guideline (Version 1.1). European Journal of Cancer, 45, 228-247.

[32] Cox, J.D., Stetz, J. and Pajak, T.F. (1995) Toxicity Criteria of the Radiation Therapy Oncology Group (RTOG) and the European Organization for Research and Treatment of Cancer (EORTC). International Journal of Radiation Oncology ${ }^{\star}$ Biology ${ }^{\star}$ Physics, 31, 1341-1346. https://doi.org/10.1016/0360-3016(95)00060-C

[33] Edge, S.B. and Compton, C.C. (2010) The American Joint Committee on Cancer: The 7th Edition of the AJCC Cancer Staging Manual and the Future of TNM. Annals of Surgical Oncology, 17, 1471-1474. 
[34] Zimmermann, M.B. and Hurrell, R.F. (2007) Nutritional Iron Deficiency. The Lancet, 370, 511-520. https://doi.org/10.1016/S0140-6736(07)61235-5

[35] Patel, KV. (2008) Epidemiology of Anemia in Older Adults. Seminars in Hematology, 45, 210-217. https://doi.org/10.1053/j.seminhematol.2008.06.006

[36] Blitzer, P., Wang, C. and Suit, H. (1984) Blood Pressure and Hemoglobin Concentration: Multivariate Analysis of Local Control after Irradiation for Head and Neck Cancer. International Journal of Radiation Oncology ${ }^{\star}$ Biology ${ }^{\star}$ Physics, 10, 98. https://doi.org/10.1016/0360-3016(84)90699-0

[37] Forastiere, A.A., Berkey, B., Maor, M., et al. (2001) Phase III Trial to Preserve the Larynx: Induction Chemotherapy and Radiotherapy versus Concomitant Chemoradiotherapy versus Radiotherapy Alone. Intergroup Trial R91-11. Proceedings of the American Society of Clinical Oncology, 20, 2a.

[38] Calais, G., Alfonsi, M., Bordet, E., et al. (2001) Radiation (RT) Alone versus RT with Concomitant Chemotherapy (CT) in Stages III and IV Oropharynx Carcinoma: Final Results of the 94-01 GORTEC Randomized Study. International Journal of Radiation Oncology ${ }^{\star}$ Biology ${ }^{\star}$ Physics, 51, 1-2. https://doi.org/10.1016/S0360-3016(01)01826-0

[39] Welsh, L., Panek, R., Riddell, A., Wong, K., Leach, M.O., Tavassoli, M., et al. (2017) Blood Transfusion during Radical Chemo-Radiotherapy Does Not Reduce Tumor Hypoxia in Squamous Cell Cancer of the Head and Neck. British Journal of Cancer, 116, 28-35. https://doi.org/10.1038/bjc.2016.386

[40] Montgomery, J., Syed, M.I., Rana, I., Singh, J. and Clark, L.J. (2010) Hemoglobin Monitoring in Head and Neck Cancer Patients Undergoing Radiotherapy. Annals of Otology, Rhinology \& Laryngology, 119, 472-475. https://doi.org/10.1177/000348941011900708

[41] Warde, P., O’Sullivan, B., Bristow, R.G., et al. (1998) T1/T2 Glottic Cancer Managed by External Beam Radiotherapy: The Influence of Pretreatment Hemoglobin on Local Control. International Journal of Radiation Oncology ${ }^{\star}$ Biology ${ }^{\star}$ Physics, 41, 347-353. https://doi.org/10.1016/S0360-3016(98)00062-5

[42] Tucker, J., Hixson, W. and Kim, R. (1996) Pretreatment Hemoglobin in Early Stage Glottic Cancer: Red Flag or Red Herring? International Journal of Radiation Oncology ${ }^{\star}$ Biology ${ }^{\star}$ Physics, 36, 336. https://doi.org/10.1016/S0360-3016(97)85696-9

[43] Canaday, D.J., Regine, W.F., Mohiuddin, M., et al. (1999) Significance of Pretreatment Hemoglobin Level in Patients with T1 Glottic Cancer. Radiation Oncology Investigations, 7, 42-48. https://doi.org/10.1002/(SICI)1520-6823(1999)7:1<42::AID-ROI6>3.0.CO;2-W

[44] Sakata, K., Aoki, Y., Karasawa, K., et al. (1994) Radiation Therapy in Early Glottic Carcinoma: Uni- and Multivariate Analysis of Prognostic Factors Affecting Local Control. International Journal of Radiation Oncology ${ }^{\star}$ Biology ${ }^{\star}$ Physics, 30, 1059-1064. https://doi.org/10.1016/0360-3016(94)90310-7

[45] Harrison, L.B., Chadha, M., Hill, R.J., et al. (2002) Impact of Tumor Hypoxia and Anemia on Radiation Therapy Outcomes. The Oncologist, 7, 492-508.

https://doi.org/10.1634/theoncologist.7-6-492

[46] Denis, F., Garaud, P., Bardet, E., et al. (2004) Final Results of the 94-01 French Head and Neck Oncology and Radiotherapy Group Randomized Trial Comparing Radiotherapy Alone with Concomitant Radiochemotherapy in Advanced-Stage Oropharynx Carcinoma. Journal of Clinical Oncology, 22, 69-76.

https://doi.org/10.1200/JCO.2004.08.021 